\title{
A KNOWLEDGE-BASED SYSTEM FOR THE AUTOMATIC CHRONOPOTENTIOMETRIC ELUCIDATION OF ELECTRODE REACTION MECHANISMS
}

\author{
M. BOS*, E. HOOGENDAM and W.E. VAN DER LINDEN \\ Department of Chemical Technology, Technical University Twente, P.O. Box 217, 7500 AE \\ Enschede (The Netherlands)
}

(Received 11th November 1987)

\section{SUMMARY}

A knowledge-based system for the elucidation of electrode reaction mechanisms based on chronopotentiometric experiments is described. The system runs the diagnostic experiments and uses the results in the reasoning process. New mechanistic knowledge can be added directly to its knowledge base in the form of production rules. The system is fully modular and its domainspecific modules can easily be changed for application to other electrochemical techniques. Correct operation of the system is demonstrated with the familiar reduction mechanisms of cadmium(II), zinc(II), cystamine and cinnamaldehyde.

Research in the field of automation in analytical chemistry has introduced the tools for operating a laboratory for routine analysis automatically. Computers and their associated software play a major role in this respect. Table 1 shows the kind of hardware and software that is now available for the various

\section{TABLE 1}

Tools for the automation of routine analyses

\begin{tabular}{|c|c|c|}
\hline & Hardware & Software \\
\hline Work lists & Minicomputers & $\begin{array}{l}\text { Laboratory information management } \\
\text { systems }\end{array}$ \\
\hline Sample preparation & Robots & Robot control languages \\
\hline Measurements & On-line $\mathrm{mini} / \mathrm{microcomputers}$ & Data acquisition and control software \\
\hline Data processing & Mini/microcomputers & $\begin{array}{l}\text { State/parameter estimation; curve fitting; } \\
\text { pattern recognition; expert systems }\end{array}$ \\
\hline $\begin{array}{l}\text { Report generation } \\
\text { Quality control } \\
\text { Filing of data }\end{array}$ & Minicomputers & $\begin{array}{l}\text { Laboratory information management } \\
\text { systems }\end{array}$ \\
\hline
\end{tabular}


steps. In routine analysis, a fixed choice of the techniques used for sample preparation, measurements and data processing is generally made. The sample routing problem can be solved by queueing theory. The other steps rely on dedicated software which either is available or can be developed with existing procedures. For non-standard samples, the situation is quite different. Here the handling of the samples require either the development of a completely new method or the modification of an existing one. The stages of development that can be distinguished are given in Table 2 together with the tools that can support these activities.

A lot of attention has already been given to the generalization of software for the automation and optimization of analytical procedures; standard building blocks for the development of the software for data acquisition, data handling and optimization are readily available in the form of subroutines in various laboratory computer software packages. The problems in the final stages of the development of new analytical procedures are generally not particular to the analytical technique, so that they can be solved by using these general tools. The first three steps in the development of new analytical procedures have the common feature that they all rely heavily on "nonprocedural" knowledge. Because of its descriptive nature, this knowledge cannot be expressed in standard mathematical equations and is normally provided by experienced analytical chemists. Recent analytical interest in knowledge-based systems, computer programs from the domain of artificial intelligence, has been focussed on the application of these tools in routine analysis $[1,2]$.

The aim of this paper is to demonstrate that a knowledge-based system can also be useful in the development of new analytical procedures. Attention is focussed on automation of the third stage, i.e., the actual development of an analytical procedure based on an electroanalytical technique. In electroanalysis, a single computerized instrument can be used for almost any of the numerous available measuring techniques. To establish which technique is best for a given analytical problem is a considerable problem and generally requires detailed knowledge of the mechanism of the electrode reaction. For new compounds, this knowledge can only be obtained experimentally. Decisions as to

\section{TABLE 2}

Stages in the development of new analytical procedures

\begin{tabular}{ll}
\hline Step in process & Automation technique \\
\hline Choice of analytical technique & Knowledge-based system \\
Choice of sample preparation technique & Knowledge-based system \\
Design of analytical procedure & Knowledge-based system \\
Automation of procedure & Data acquisition and data processing software \\
Optimization of procedure & Optimization software \\
\hline
\end{tabular}


which experiments are to be done with the various electrochemical techniques are based on the experience of the electrochemist and are re-evaluated each time new experimental information becomes available. The repertoire of methods generally used for this purpose includes cyclic voltammetry, various forms of polarography, chronopotentiometry and rotating ring/disk voltammetry.

In this paper, an attempt is made to automate the elucidation of electrode reaction mechanisms with a system in which the knowledge of chronopotentiometry as collected by Reinmuth [3] is used in the form of a rule-based expert system. Chronopotentiometry was chosen as a start because the knowledge in this field is available as a concise set of rules. The computer language FORTH was used to program the system. This language is particularly suitable for laboratory automation and can also provide the complicated data structures that are needed to represent the chronopotentiometric knowledge in the form of rules, facts and hypotheses. This language also provided the means to the second goal of this investigation, viz., the integration of expert system software and software for controlling the experiment, data acquisition and data handling. It also allows later expansion of the system to other electrochemical techniques.

\section{THE CHROPO SYSTEM}

The CHROPO system is a modular, rule-based and relatively simple expert system (see Fig. 1). Its major feature is that it incorporates routines that can run and evaluate chronopotentiometric experiments. These routines provide the knowledge that the expert system part needs for the elucidation of electrode reaction mechanisms. There is a module that accepts operator input, but in normal operation the system chooses the experiments, runs them and finally decides on the mechanism all by itself.

In the design of the system, such attention has been given to the separation

USRR INTERPACE $\quad$ KNOULEDGE BASB

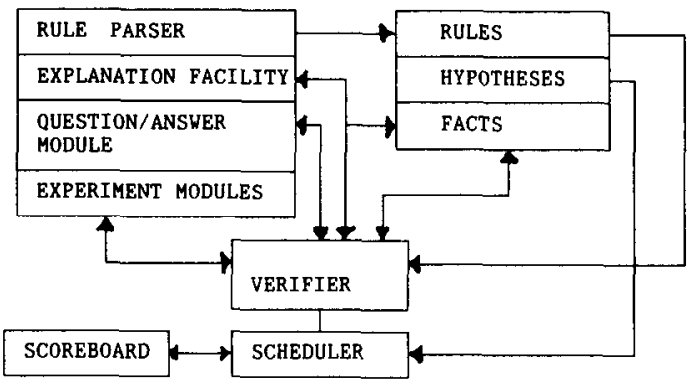

INFERENCE ENGINE

Fig. 1. Schematic diagram of CHROPO system. 
of problem-dependent and problem-independent parts in different modules. The knowledge base and the experimental modules are problem-specific; the remainder of the system can be used for any technique.

\section{The chronopotentiometry modules}

The system for identification of reaction mechanisms given by Reinmuth [3] is based on five diagnostic criteria that can be determined from chronopotentiograms measured with current reversal at various current values and various concentrations of the compound of interest. For each of these criteria, a module was developed that performs the experiments and determines the value of a criterion. The prerequisite condition that the electrode process should be diffusion-controlled is verified by a module that checks the constancy of the product of the current and the square root of the transition time $\left(i_{0} \tau^{1 / 2}\right)$.

Statistics are used to decide whether the criteria are satisfied within experimental errors. The system cannot decide on the number of electrons involved in the electrochemical step. This information must now be provided by the user, but in further developments it will be supplied by another automated technique. In the rules, the values of the criteria that are dependent on this number of electrons are normalized to a one-electron transfer. The data-handling parts of the experiment routines comply with this requirement.

Statistics. For four of the criteria, viz., the slope of the linear logarithmic plot (vide infra), $\delta E_{1 / 4} / \delta \ln i_{0}$ and $\delta E_{1 / 4} / \delta \ln C^{*}$ and the product $i_{0} \tau^{1 / 2}$, the parameter of interest is the value of the slope of a linear plot. The null hypothesis that the experimental slope equals a diagnostic value is tested by using the least-squares method and the Student $t$ tables. Linearity of the logarithmic plot of the potential versus $\left(\tau^{1 / 2}-t^{1 / 2}\right) / \mathrm{t}^{1 / 2}$ or versus $\left(\tau^{1 / 2}-t^{1 / 2}\right)$ is tested by using the Fisher test in which the variance of the potential data around the regression line is divided by the variance caused by the $A / D$ converter, and checking whether this value is below 1.4, the limiting value for a high number of data points.

For the value of the ratio of the reverse and forward transition time, $\tau_{\mathrm{r}} / \tau_{\mathrm{f}}$, the test value is checked against the $2 \sigma$ interval around the experimentally found value.

Evaluation of chronopotentiograms. Several methods for the determination of the transition time $\tau$ and the potential at a quarter of the transition time $\left(E_{1 / 4}\right)$ which correct for the charging of the electrical double-layer capacitance were summarized by Bos [4]. Bos recommended the procedure given by de Vries [5], which uses the construction of Delahay and Mattax [6] with a controlled starting potential. This method is implemented in the CHROPO system as depicted in Fig. 2. The tangents $l$ and $m$ are drawn in the points $\left(l_{1}, l_{2}\right)$ and $\left(m_{1}, m_{2}\right)$, where the first derivative of the curve has a local maximum. The line $n$ is drawn at $1 / 4$ of the distance from $l$ to $m$. The intersection of $n$ with the potential/time curve gives the value of $E_{1 / 4}$. The transition time is found 


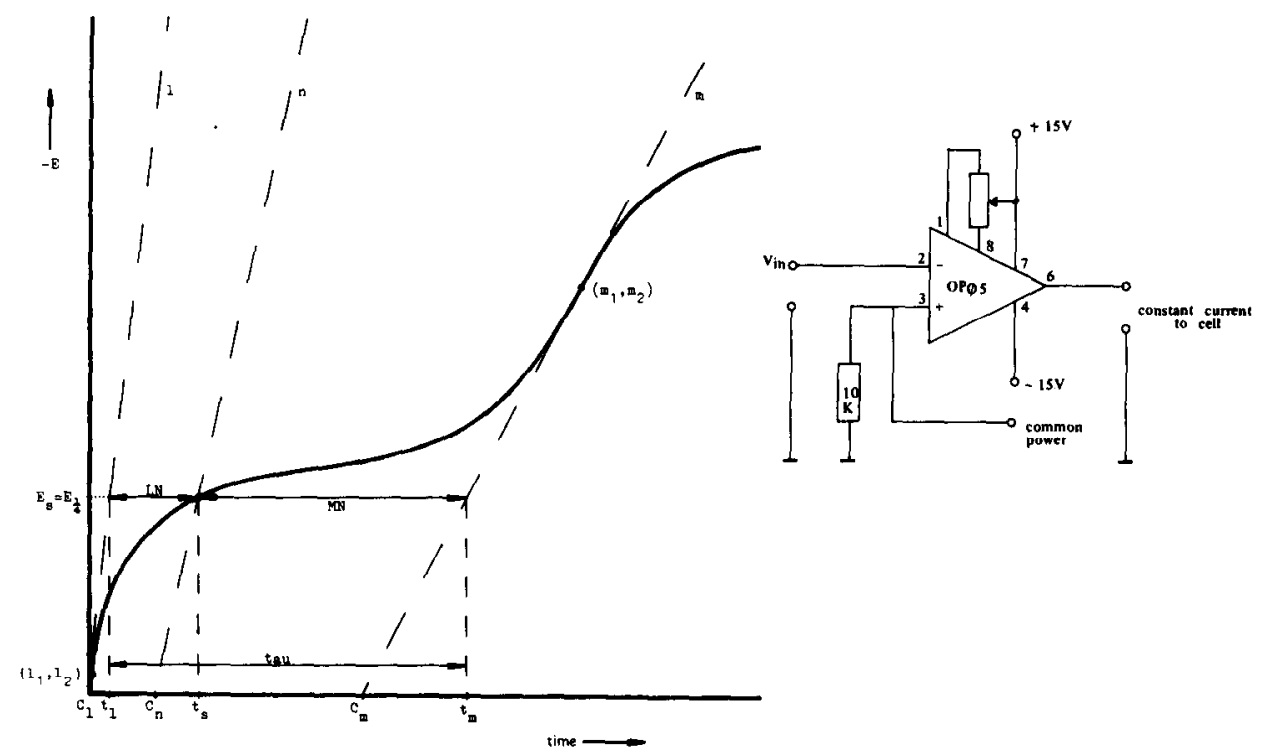

Fig. 2. Evaluation of $E_{1 / 4}$ and transition time from chronopotentiograms.

Fig. 3. Schematic diagram of constant-current source.

as the length that is cut off from a horizontal line at $E_{1 / 4}$ by $l$ and $m$. This method is only applicable if the experiment starts at a potential that is 200 / (number of electrons) $\mathrm{mV}$ positive with respect to $E_{1 / 4}$. The required starting potential is found in a preliminary run.

The ratio of the reverse to forward transition time also requires a preliminary run to establish the time limit for the forward electrolysis. The experiment modules automatically take care of these preliminary runs.

\section{The expert system}

The knowledge base. The complete CHROPO system consists of three main parts: the knowledge base, the inference engine and the user interface. The knowledge base contains the diagnostic table from Reinmuth [3] in the form of rules and the various mechanisms as hypotheses. At the start of the run, no facts are known. This data base is gradually filled by the experiment modules while the procedure progresses and by the reasoning mechanism of the inference engine.

The basic semantic unit in the knowledge base is the associative triple [7], which is used to describe the facts and the hypotheses. It consists of three elements: the attribute of an object, the object and the value of the attribute of the object. The rule syntax is given in Table 3 in Backus Naur form. The ruleparser module converts rules of the correct syntax to an efficient internal rep- 
TABLE 3

Backus Naur form of rule syntax

\begin{tabular}{ll}
\hline$<$ rule $>$ & $::=\{<$ if clause $><$ then clause $>\}$ \\
<if clause $>$ & $::=\{$ IF $<$ clause $>\backslash$ OR $<$ clause $>\backslash\}$ \\
<clause $>$ & $::=\{<$ antecedent $>\backslash\}+$ \\
<then clause $>$ & $::=\{$ THEN $\{<$ consequent $>\backslash\}+\backslash\}$ \\
$<$ antecedent $>$ & $::=<$ associative triple $>\vee$ NOT $<$ associative triple $>\}$ \\
$<$ consequent $>$ & $::=<$ associative triple $>\vee N O T<$ associative triple $>\}$ \\
$<$ associative triple $>$ & $::=\{<$ attribute $><$ object $><$ value $>\}$ \\
<attribute $>$ & $::=<$ character string $>$ \\
$<$ object $>$ & $::=<$ character string $>$ \\
$<$ value $>$ & $::=<$ character string $>\vee<$ integer value $>\vee<$ < integer value $>\vee$ \\
& $><$ integer value $>$
\end{tabular}

resentation. It uses the backslash ( $\backslash$ ) delimiter and the keywords IF, OR, NOT and THEN to analyze the rules. Table 4 gives the coding of mechanism 1 from the Reinmuth table as an example (a simple rapid reversible reduction).

The data base for the facts contains the associative triples that are known to be either true or false (internally represented by the values 1 and -1 ). If an associative triple cannot be found in the facts data base, this means that the corresponding fact is still unknown.

The data base for the hypotheses simply consists of a list of the associative triples representing the hypotheses.

The inference engine. The inference engine consists of three modules: the verifier, the scheduler and the scoreboard. Its heart is the verifier which is implemented as a recursive backward-chaining mechanism. The most important routines in the verifier are CHECKFACT and TRY_RULE. CHECKFACT tries to evaluate a hypothesis using the database of facts. TRY_RULE tries to evaluate the hypothesis by finding a rule that has the hypothesis as a consequence and by evaluating the antecedents of this rule. In this process, it calls the verifier recursively. Finally, a stage is reached where an antecedent for which there is no rule has to be evaluated. The verifier then calls in an experiment routine that provides the logical value for this antecedent. If there is no such routine, or if the routine has been switched off for testing purposes, the verifier asks the user for the required information.

The CHECKFACT routine succeeds if the data base of facts contains a fact with the same attribute and the same object as the hypothesis. If its value also matches the value of the hypothesis, the job of the verifier is completed by returning the value true or false that is stored for the fact. If the values of the hypothesis and the fact do not match, the logical value of the hypothesis is evaluated by using the rule that an attribute/object pair can have only one value that excludes all others. For integer numerical data within the value 
Coded rules describing rapid reversible reaction mechanism (M1)

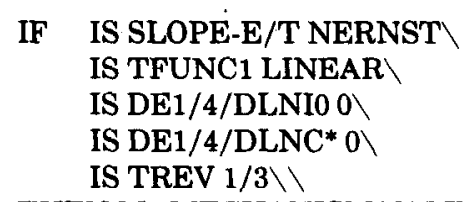

THEN M1 MECHANISM VALID $\backslash \backslash$

IF VALUENORM SLOPE-E/T $>55 \backslash$

VALUENORM SLOPE-E/T $<65 \backslash \backslash$

THEN IS SLOPE-E/T NERNST \\}

IF IS LINEAR-E/T-FUNC $1 \backslash \backslash$

THEN IS TFUNC1 LINEAR \\}

IF VALUENORM DE1/4/DLNI0 $0 \backslash \backslash$

THEN IS DE1/4/DLNI0 0 \\

IF VALUENORM DE1/4/DLNC* $0 \backslash \backslash$

THEN IS DE1/4/DLNC* $0 \backslash$ !

IF VALUE TREV $>31 \backslash$

VALUE TREV <35 \\

THEN IS TREV $1 / 3 \backslash \backslash$

ranges of their associative triples, hypotheses are allowed to have the larger than $(>)$ or smaller than $(<)$ operator preceding the numerical value. They are evaluated by using signed 16-bit integer arithmetic for the comparison.

The TRY_RULE routine incorporates generation of intermediate propositions. New facts established by applying a rule are added to the data base of facts, thereby increasing efficiency because a subsequent evaluation of the consequence of the rule now succeeds directly via CHECKFACT.

A simplified description of the VERIFY routine in pseudo-code is given in Table 5.

The scheme for controlling which hypothesis to present to the verifier is implemented via the scheduler/scoreboard parts of the inference engine. It looks for that hypothesis which needs a minimum of experiments to establish its logical value. If a hypothesis is rejected, the scoreboard is updated to show the number of experiments needed for each of the remaining hypotheses.

The user interface. The user interface enables the user to enter the rules in the knowledge base and offers an explanation facility. Rules can be added to the knowledge base directly from keyboard, but a more convenient way is to use the system editor to prepare a RULES file. This file can be read by the rule-parser. The explanation facility shows the user the rules in the chain of reasoning made by the system, and presents the facts that are known to the system at a given moment. The question/answer module is activated by the verifier if it needs external information that cannot be provided by one of the 
TABLE 5

Pseudo-code for simplified recursive KERIFY routine

IF

hypothesis is in data base of facts

THEN

take result from data base of facts

ELSE

IF

there is a rule with the hypothesis as a consequence

THEN

VERIFY antecedents of this rule and take result from evaluation of this rule

ELSE

IF

there is an experiment-running routine associated with the

THEN hypothesis

run the associated experiment and take result from

ELSE evaluation of experiment

ask the operator

ENDIF

ENDIF

ENDIF

experimental modules. This will not happen during a normal run of the program, but it is essential in the development phase when new rules are added and have to be tested.

\section{EXPERIMENTAL}

\section{Chemicals and equipment}

Potassium chloride, cadmium sulphate and zinc sulphate (Merck, Suprapur grade) were used as received. Cinnamaldehyde and cystamine hydrochloride (Fluka) were also used without further purification. All solutions were prepared with deionized water which was filtered through Millipore Q-II filters. Nitrogen of very pure grade (Hoekloos) was used to expel oxygen from the samples. The mercury was polarographic grade (Drijfhout).

The measuring cell consisted of a glass vessel with a mercury pool (area 110 $\mathrm{mm}^{2}$ ) as the working electrode and a platinum wire as the counter electrode. The working electrode potential was measured against a calomel electrode 


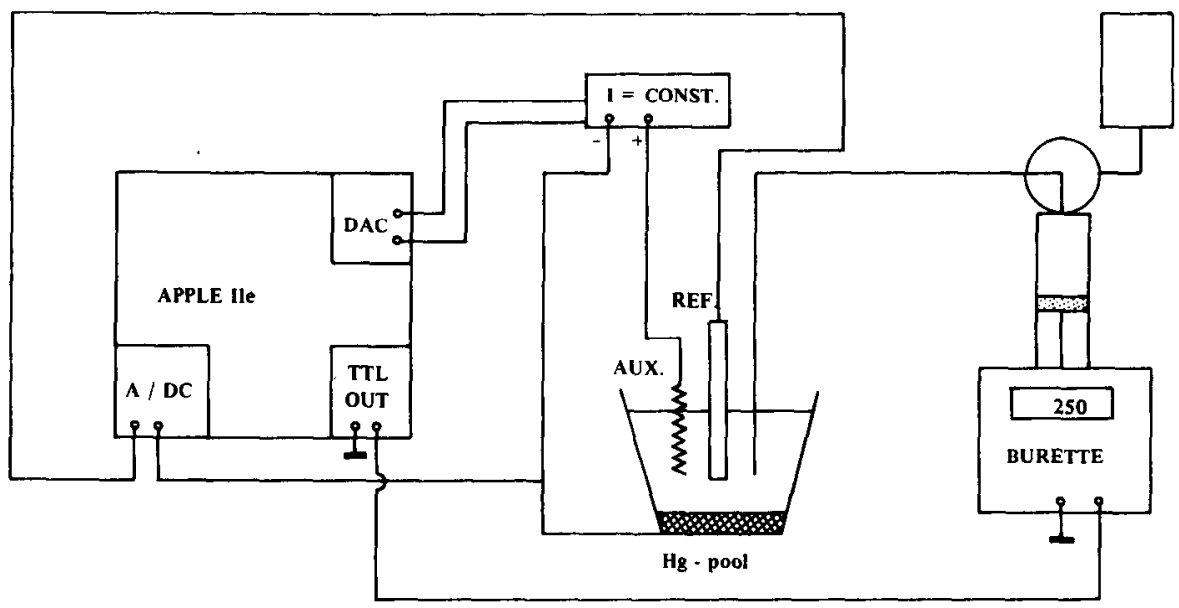

Fig. 4. Equipment for computerized chronopotentiometry.

(Metrohm) which was filled with $3 \mathrm{M}$ potassium chloride. The electrode mounting provided tubes for flushing the cell contents and the space above the sample with nitrogen.

Chronopotentiometric operation of the cell was automated with an Apple IIe computer equipped with a Digiloc 12 -bit A/D-converter (range $\pm 1 \mathrm{~V}$ ) and a Kronemuis 12-bit D/A-converter (range $0-10 \mathrm{~V}$ ). Cell current was applied by a home-made constant-current source. The control voltage for this constantcurrent source was delivered by the $\mathrm{D} / \mathrm{A}$ converter in the computer; $10-\mathrm{V}$ control voltage produces a current of $1.000 \mathrm{~mA}$. A schematic diagram of the constant-current source is given in Fig. 3. Additions of the electroactive sample to the cell to change its concentration were made by a Radiometer ABU-12 autoburette which was adapted to computer control in its fixed-increment mode via a TTL reed relay. Activating this relay during a $100-\mathrm{ms}$ interval guarantees a delivery of $0.001 \mathrm{ml}(1 / 250$ of total burette volume). A schematic diagram of the equipment is given in Fig. 4.

\section{Procedures}

Before a run is started, oxygen is expelled from the sample in the cell by bubbling with nitrogen for $20 \mathrm{~min}$. The protocol for the computerized recording of a chronopotentiogram comprises the following steps: (1) maintain the working electrode at the rest potential (fixed by operator setting) for $40 \mathrm{~s}$ during which nitrogen is bubbled through the solution; (2) maintain the working electrode at the prepotential (at $200 \mathrm{mV} /$ number of electrons) with the sample solution at rest and under nitrogen blanket for $30 \mathrm{~s}$ (control of the rest potential and the prepotential is effected by a proportional control algorithm that adjusts the cell current based on the deviation between the set point and 
the measured value of the potential of the working electrode); (3) record the actual chronopotentiogram (the first part of the curve is computer-recorded at a sampling interval of $10 \mathrm{~ms}$ during a period of $400 \mathrm{~ms}$; thereafter the sampling frequency is adjusted in such a manner that 100-200 data points are gathered until the potential reaches a value $400 \mathrm{mV}$ cathodic of the $E_{1 / 4}$ value so that the transition is fully recorded); (4) restore the working electrode at the rest potential under nitrogen stirring, in order to redissolve any amalgam formed and to prepare the cell for the next run; (5) evaluate the chronopotentiogram by the procedure outlined above to calculate the transition time $\tau$ and the value of $E_{1 / 4}$.

For each of the diagnostic criteria, a measuring scheme was developed in which this basic protocol is used so many times that statistics can be applied to ascertain the confidence limits of the quantities to be evaluated. The following measuring schemes were used.

Constancy of the product $\mathrm{i}_{0} \tau^{1 / 2}$. A prerequisite for the validity of the diagnostic criteria given by Reinmuth [3] is diffusion control of the electrode reaction. The CHROPO system always starts by verifying this condition, for which purpose it measures the constancy of the product of the current and the square root of the transition time at five different values of the current. At each current, triplicate chronopotentiograms are run. The five different currents are chosen in such a way that their corresponding transition times are evenly divided in the range from 5-25 s. They are calculated from the estimate of the product of $i_{0} \tau^{1 / 2}$ that is obtained in the preliminary runs. The values obtained for $i_{0} \tau^{1 / 2}$ in this run are used to test the statistical null hypothesis that $i_{0} \tau^{1 / 2}$ is a constant. The average of these values is used to update the original estimate of $i_{0} \tau^{1 / 2}$ to improve the accuracy with which following measuring schemes can be set up. The same averaging procedure is applied to the $E_{1 / 4}$ values obtained in this series of experiments to update the $E_{1 / 4}$ estimate from which the prepotential for subsequent runs is calculated.

The ratio $\tau_{r} / \tau_{f}$. The ratio of the reversal transition to the forward transition is determined from five replica experiments, each comprising the following steps: (1) maintain the working electrode at the rest potential for $40 \mathrm{~s}$ during which nitrogen is bubbled through the cell; (2) maintain the working electrode at the prepotential for $30 \mathrm{~s}$ with the solution at rest; (3) apply a constant current during $12 \mathrm{~s}$ (the value of this current is chosen to give a transition time of $15 \mathrm{~s}$ ); (4) reverse the current; (5) measure the working electrode potential at 40 -ms intervals until well past the backward transition; (6) switch the nitrogen stream to stir the solution; (7) restore the working electrode to the rest potential for $30 \mathrm{~s} ;(8)$ calculate the ratio $\tau_{\mathrm{r}} / \tau_{\mathrm{f}}$.

After five replicate measurements have been made, the confidence limit for the ratio $\tau_{\mathrm{r}} / \tau_{\mathrm{f}}$ is calculated.

The ratio $\delta \mathrm{E}_{1 / 4} / \delta \ln \mathrm{i}_{0}$. For this criterion, the measurements from the $i_{0} \tau^{1 / 2}$ 
constancy test are used. Linear regression is used to obtain the confidence limit of the slope of the plot of the $E_{1 / 4}$ values versus $\ln i_{0}$.

The ratio $\delta \mathrm{E}_{1 / 4} / \delta \ln \mathrm{C}^{*}$. At five different concentrations of the electroactive component; triplicate chronopotentiograms are recorded and evaluated, all at the same cell current. The current and the concentrations are chosen to give transition times evenly divided between 5 and $25 \mathrm{~s}$. The change in concentration is effected by computer-controlled operation of the burette. Linear regression is used to obtain the confidence limit of the slope of the plot of the $E_{1 / 4}$ values versus $\ln C^{*}$.

Linear $\log$ plots. Linear $\log$ plots of $E$ versus $\log \left[\left(\tau^{1 / 2}-t^{1 / 2}\right) / t^{1 / 2}\right]$ or versus $\log \left(\tau^{1 / 2}-t^{1 / 2}\right)$, etc., also serve as criteria. To obtain the data a chronopotentiogram is run at a current to give a transition time of $22 \mathrm{~s}$. Linear regression is used to obtain the log plots. Linearity of the plots and the confidence limits of the slopes of these plots are evaluated with the statistics given above.

\section{RESULTS AND DISCUSSION}

The reduction of cadmium(II) ions in $0.1 \mathrm{M} \mathrm{KCl}$ follows the first reaction mechanism from the Reinmuth [3] table, i.e., a rapid reversible reduction. The CHROPO system correctly concludes this by consecutively running and evaluating the following modules: (1) constancy of the product $i_{0} \tau^{1 / 2},(2) \delta E_{1 / 4} /$ $\delta \ln i_{0},(3)$ current reversal, (4) $\delta E_{1 / 4} / \delta \ln C^{*}$, and (5) linear plot of $E$ versus $\log \left[\left(\tau^{1 / 2}-t^{1 / 2}\right) / t^{1 / 2}\right]$. Modules 1 and 2 use the same set of measurements. The complete results are summarized in Table 6. The criteria for mechanism 1 are all satisfied very well. It should be noted that, although mechanistically this reversible rapid reduction is the simplest one, its criteria are the hardest to meet because all require a specified value and not a range as for most of the other possible mechanisms. Another reason why this mechanism is a good test case is that it relies on all criteria, without exception.

For the reduction of zinc(II) ions in $0.1 \mathrm{M} \mathrm{KCl}$, the CHROPO system decides on an irreversible mechanism, as the $E_{1 / 4}$ is found to be dependent on the current as well as on the bulk concentration of zinc(II). Moreover, a different transformation of the time axis is needed to obtain a linear $E$ plot. The results are summarized in Table 6.

For cystamine, CHROPO also finds an irreversible reduction mechanism. In contrast to the zinc (II) system, no transition is found when current reversal is applied. The results are given in Table 7.

The reduction of aromatic aldehydes at low $\mathrm{pH}$ values is known [8] to proceed via a one-electron transfer step followed by dimerization. CHROPO correctly decides on this mechanism but cannot distinguish between a slow or an irreversible dimerization step (entries 13 and 15 in the Reinmuth table) as the criteria have the same values for both mechanisms. The results for this system are also given in Table 7 . 
TABLE 6

Chronopotentiometry of $\mathrm{Cd}(\mathrm{II})$ and $\mathrm{Zn}$ (II) in $0.1 \mathrm{M} \mathrm{KCl}$

\begin{tabular}{|c|c|c|c|c|c|c|c|}
\hline $\begin{array}{l}i_{0} \\
(\mu \mathrm{A})\end{array}$ & $\begin{array}{l}E_{1 / 4} \\
(\mathrm{mV})\end{array}$ & $\tau$ & $i_{0} \tau^{1 / 2}$ & $\begin{array}{l}\text { Conc. } \\
\left(10^{-4} \mathrm{M}\right)\end{array}$ & $\begin{array}{l}E_{1 / 4} \\
(\mathrm{mV})\end{array}$ & $\begin{array}{l}\tau \\
(\mathbf{s})\end{array}$ & $C^{*} / \tau^{1 / 2}$ \\
\hline \multicolumn{8}{|c|}{$\operatorname{Cadmium}(I I)$} \\
\hline 241 & -611 & 4.488 & 510.6 & 4.78 & -608 & 6.56 & 1.87 \\
\hline 170 & -609 & 8.490 & 495.3 & 5.75 & -607 & 8.56 & 1.96 \\
\hline 139 & -609 & 12.666 & 494.7 & 6.92 & -608 & 12.70 & 1.94 \\
\hline 120 & -609 & 17.273 & 498.7 & 7.94 & -608 & 16.15 & 1.97 \\
\hline 107 & -608 & 21.939 & 501.1 & 8.70 & -608 & 19.37 & 1.97 \\
\hline \multicolumn{8}{|c|}{$\operatorname{Zinc}(I I)$} \\
\hline 133 & -1044 & 5.038 & 298.5 & 4.95 & -1034 & 7.522 & 1.80 \\
\hline 94 & -1037 & 9.976 & 296.9 & 5.94 & -1032 & 10.103 & 1.87 \\
\hline 77 & -1032 & 15.323 & 301.4 & 7.15 & -1029 & 13.912 & 1.92 \\
\hline 66 & -1028 & 20.614 & 299.7 & 8.21 & -1026 & 18.119 & 1.93 \\
\hline 59 & -1024 & 26.374 & 303.0 & 9.00 & -1024 & 21.627 & 1.94 \\
\hline \multicolumn{8}{|c|}{$\begin{array}{l}\text { Cadmium(II) } \\
\mathrm{d}\left(i_{0} \tau^{1 / 2}\right) / \mathrm{d} i_{0}=0.08 \pm 0.23 . \mathrm{d} E_{1 / 4} / \mathrm{dlog} i_{0}=-6.5 \pm 7.9 . \mathrm{d} E_{1 / 4} / \mathrm{dlog} C^{*}=-1.5 \pm 15.0\end{array}$} \\
\hline \multicolumn{8}{|c|}{$\begin{array}{l}\text { Current reversal with } i_{0}=129 \mu \mathrm{A}: \tau_{\mathrm{r}} / \tau_{\mathrm{f}}=0.34 \pm 0.03 \\
E(\mathrm{mV}) \text { vs. } \log \left[\left(\tau^{1 / 2}-t^{1 / 2}\right) / t^{1 / 2}\right]: \text { slope }=31.8 \mathrm{mV}, \sigma^{2}=6.04\end{array}$} \\
\hline \multicolumn{8}{|c|}{$\begin{array}{l}Z \operatorname{Zinc}(I I) \\
\mathrm{d}\left(i_{0} \tau^{1 / 2}\right) / \mathrm{d} i_{0}=0.05 \pm 0.16 . \mathrm{d} E_{1 / 4} / \mathrm{d} \log i_{0}=-56.6 \pm 13.5 . \mathrm{d} E_{1 / 4} / \operatorname{dlog} C^{*}=36.1 \pm 16\end{array}$} \\
\hline \multicolumn{8}{|c|}{$\begin{array}{l}\text { Current reversal with } i_{0}=77 \mu \mathrm{A}: \tau_{\mathrm{r}} / \tau_{\mathrm{f}}=0.383 \pm 0.03 \\
E(\mathrm{mV}) \text { vs. } \log \left(\tau^{1 / 2}-t^{1 / 2}\right): \text { slope }=61.2 \mathrm{mV}, \sigma^{2}=1.60\end{array}$} \\
\hline
\end{tabular}

Although the present CHROPO system is limited to the fifteen E or EC mechanisms described by Reinmuth [3], this work shows that the use of a knowledge-based system makes it feasible to automate the complete process of electrode reaction mechanism elucidation. It also shows that it will be necessary to include other electrochemical techniques to reach this goal. The CHROPO system, for example, cannot decide on the number of electrons transferred in the electrochemical step. The main advantage of using a knowledge-based system in which the domain-specific knowledge is separated from the reasoning logic is that it can easily be extended to other required domains.

The authors express their thanks to J.M. Plantinga, who developed the statistical routines and a number of the experimental modules. 


\section{TABLE 7}

Chronopotentiometry of cystamine in $0.1 \mathrm{M}$ acetic acid/sodium acetate, and cinnamic aldehyde in $0.1 \mathrm{M}$ glycine $/ \mathrm{HCl}$ buffer $\mathrm{pH} 1.0$ and ethanol $(70 / 30, \mathrm{v} / \mathrm{v})$

\begin{tabular}{|c|c|c|c|c|c|c|c|}
\hline $\begin{array}{l}i_{0} \\
(\mu \mathrm{A})\end{array}$ & $\begin{array}{l}E_{1 / 4} \\
(\mathrm{mV})\end{array}$ & $\begin{array}{l}\tau \\
(\mathrm{s})\end{array}$ & $i_{0} \tau^{1 / 2}$ & $\begin{array}{l}\text { Conc." }^{a} \\
\left(10^{-4} \mathrm{M}\right)\end{array}$ & $\begin{array}{l}E_{1 / 4} \\
(\mathrm{mV})\end{array}$ & $\begin{array}{l}\tau \\
(\mathrm{s})\end{array}$ & $C^{*} / \tau^{1 / 2}$ \\
\hline \multicolumn{8}{|c|}{ Cystamine } \\
\hline 49.0 & -1152 & 2.610 & 79.2 & 1.82 & -1135 & 9.459 & 0.592 \\
\hline 34.0 & -1124 & 5.989 & 83.2 & 2.18 & -1132 & 14.840 & 0.566 \\
\hline 28.0 & -1113 & 7.537 & 76.9 & 2.62 & -1121 & 23.179 & 0.544 \\
\hline 24.0 & -1112 & 12.886 & 86.2 & 3.01 & -1119 & 32.090 & 0.531 \\
\hline 22.0 & -1112 & 14.363 & 83.4 & 3.30 & -1116 & 38.142 & 0.534 \\
\hline \multicolumn{8}{|c|}{ Cinnamic aldehyde } \\
\hline 132 & -733 & 4.122 & 268 & 2.38 & -774 & 5.088 & 1.05 \\
\hline 93 & -730 & 8.508 & 271 & 2.87 & -777 & 7.820 & 1.03 \\
\hline 76 & -726 & 13.495 & 279 & 3.45 & -781 & 12.190 & 0.99 \\
\hline 66 & -727 & 17.399 & 275 & 3.96 & -783 & 17.287 & 0.95 \\
\hline 59 & -726 & 21.338 & 272 & 4.33 & -778 & 21.240 & 1.04 \\
\hline \multicolumn{8}{|c|}{$\begin{array}{l}\text { Cystamine } \\
\mathrm{d}\left(i_{0} \tau^{1 / 2}\right) / \mathrm{d} i_{0}=-0.15 \pm 0.8 . \mathrm{d} E_{1 / 4} / \mathrm{dlog} i_{0}=-120 \pm 95 . \mathrm{d} E_{1 / 4} / \mathrm{dlog} C^{*}=77.8 \pm 20 .\end{array}$} \\
\hline \multicolumn{8}{|c|}{$\begin{array}{l}\text { Current reversal with } i_{0}=21 \mu \mathrm{A}: \tau_{\mathrm{r}} / \tau_{\mathrm{f}}=0.01 \pm 0.001 \\
E(\mathrm{mv}) \text { vs. } \log \left(\tau^{1 / 2}-t^{1 / 2}\right): \text { slope }=53.6 \mathrm{mV}, \sigma^{2}=6.0\end{array}$} \\
\hline \multicolumn{8}{|c|}{$\begin{array}{l}\text { Cinnamic aldehyde } \\
\mathrm{d}\left(i_{0} \tau^{1 / 2}\right) / \mathrm{d} i_{0}=-0.09 \pm 0.28 . \mathrm{d} E_{1 / 4} / \mathrm{d} \log i_{0}=-20.9 \pm 18.7 . \mathrm{d} E_{1 / 4} / \mathrm{d} \log C^{*}=-17.0 \pm 58.5\end{array}$} \\
\hline \multicolumn{8}{|c|}{$\begin{array}{l}\text { Current reversal with } i_{0}=70 \mu \mathrm{A}: \tau_{\mathrm{r}} / \tau_{\mathrm{f}}=0.01 \pm 0.001 \\
\text { No linear log plot for } E \text { vs. transformed time axis. }\end{array}$} \\
\hline
\end{tabular}

${ }^{a}$ Concentrations are $10^{-3} \mathrm{M}$ for cinnamic aldehyde.

\section{REFERENCES}

1 R.E. Dessy, Anal. Chem., 56 (1984) 1200A, 1312A.

2 J.W.A. Klaessens, G. Kateman and B.G.M. Vandeginste, Trends Anal. Chem., 4 (1984) 114.

3 W.H. Reinmuth, Anal. Chem., 32 (1960) 1515.

4 P. Bos, Ph.D. Thesis, Free University, Amsterdam, 1970.

5 W.T. de Vries, J. Electroanal. Chem., 17 (1968) 31; 18 (1968) 469.

6 P. Delahay and C.C. Mattax, J. Am. Chem. Soc., 76 (1954) 874.

7 F. Hayes-Roth, D.A. Waterman and D.B. Lenat, Building Expert Systems, Addison-Wesley, Reading, MA, 1983.

8 I.M. Kolthoff and J.J. Lingane, Polarography, Vol. 2, Interscience, New York, 1952, p. 681. 